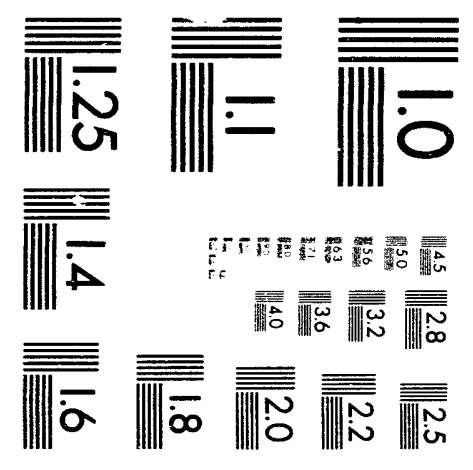



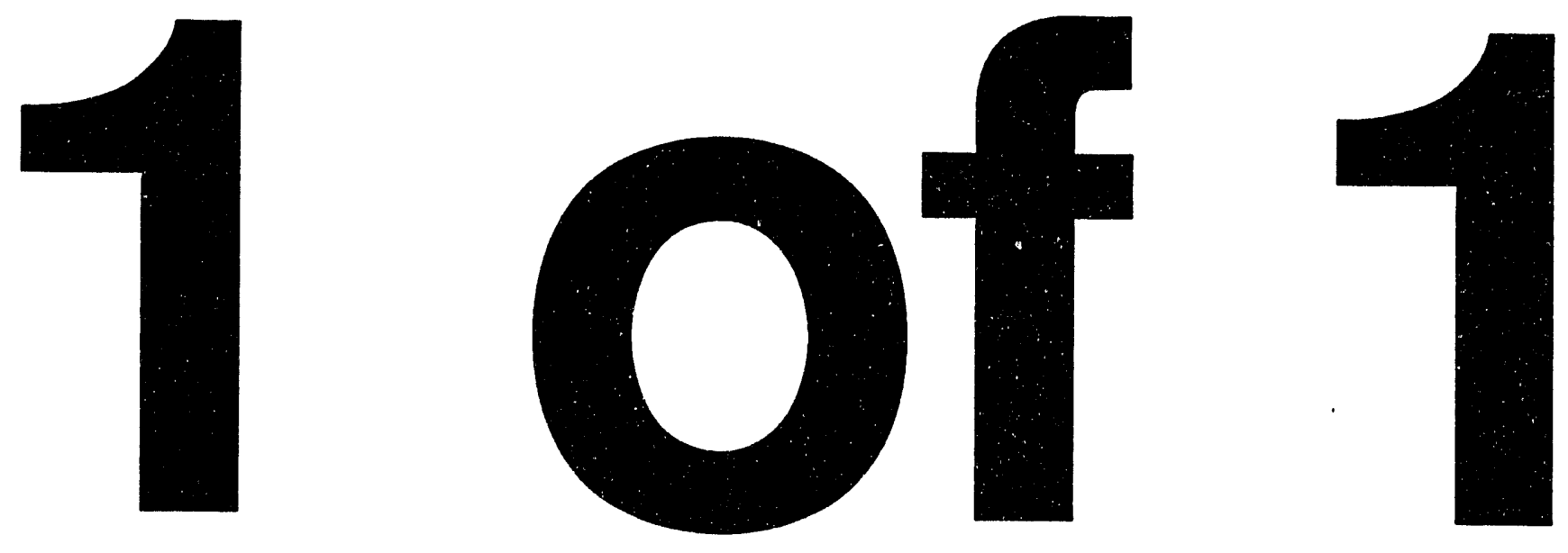
Conf-9306245--2

UCRL-JC-115309

PREPRINT

\title{
Modelling Atmospheric Turbulence Effects on Ground-Based Telescope Systems
}

\author{
L. William Bradford \\ Stanley M. Flatte \\ Claire E. Max
}

This paper was prepared for submittal to

Adaptive Optics for Astronomy

Cedex, France

June 28-July 4, 1993

September 30, 1993

This is a preprint of a paper intended for publication in a journal or proceedings. Since changes may be made before publication, this preprint is made available with the understanding that it will not be cited or reproduced without the permission of the author.

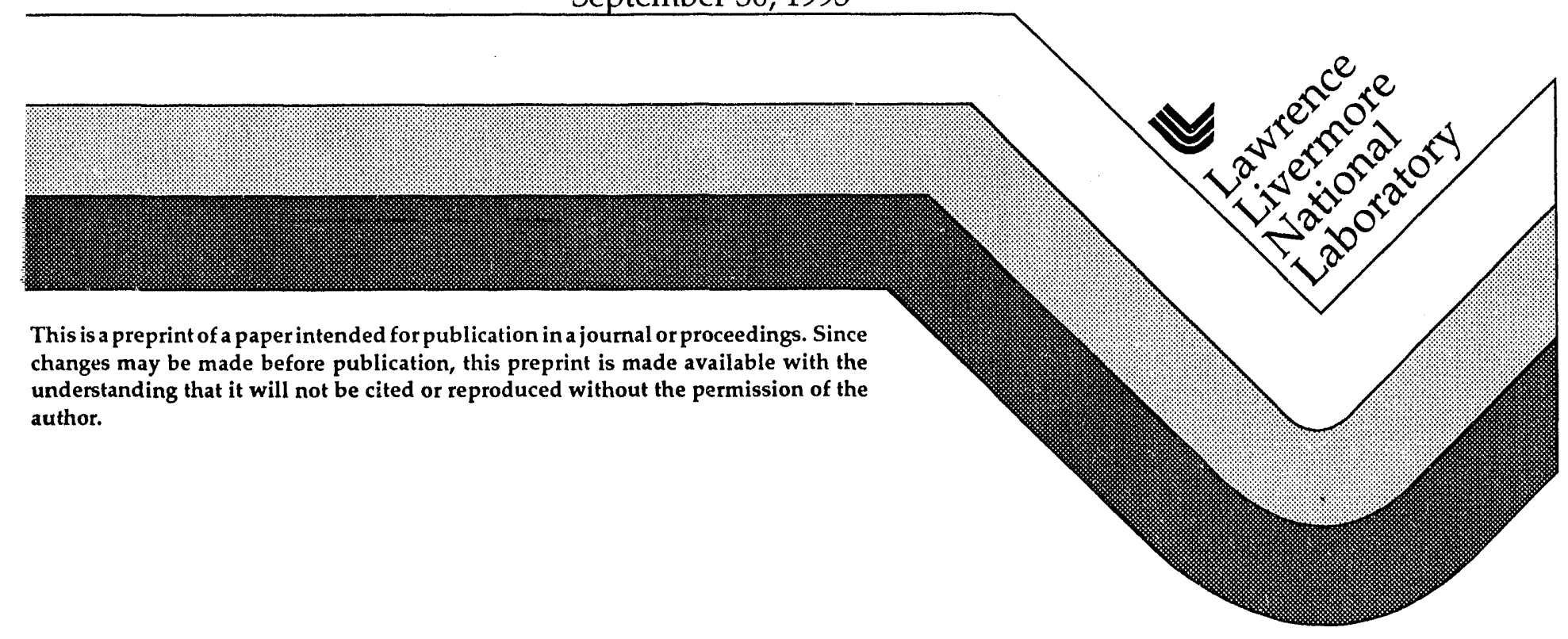




\section{DISCLAIMER}

This document was prepared as an account of work sponsored by an agency of the United States Government. Neither the United States Government nor the University of California nor any of their employees, makes any warranty, express or implied, or assumes any legal liability or responsibility for the accuracy, completeness, or usefulness of any information, apparat us, product, or process disclosed, or represents that its use would not infringe privately owned rights. Reference herein to any specific commercial products, process, or service by trade name, trademark, manufacturer, or otherwise, does not necessarily constitute or imply its endorsement, recommendation, or favoring by the United States Government or the University of California. The views and opinions of authors expressed herein do not necessarily state or reflect those of the United States Government or the University of California, and shall not be used for advertising or product endorsement purposes. 


\title{
MODELLING ATMOSPHERIC TURBULENCE EFFECTS ON GROUND-BASED TELESCOPE SYSTEMS
}

\author{
L. WILLIAM BRADFORD and STANLEY M. FLATTE \\ Physics Depurtment \\ University of California at Santa Cruz \\ and \\ CLAIRE E. MAX \\ Instilute of Geophysics and Planelary Physirs \\ Lawrence Livermore National Laboratory
}

\begin{abstract}
Questions still exist concerning the appropriate model for turbulence-induced phase fluctuations seen in ground-liaved eelescopes. Bester et al. used a particuliur observable (slope of the Allan variance) with an infrared interferometer in ai altempt to distinguish models. We have calculated that observable for fiolmogorov and "random walk" models with a varicty of ouster sciales ind allitude-dependent turbulence and wind velecity. We have found liat clear distinction bet wer:s models rectuires good dialn un lise vertical distribution of wind and turbulence. Furthernore, measurenents at time sepiarations of order 60 s are necessary lo distinguish the "random walk" model from the kolmogorov model.
\end{abstract}

Key words: almospheric turbulence, outer scale, Kolmogorov spectrum.

\section{Introduction}

Other lectures in this volume, particularly those of Fried and Roddier, have covered the cffects of atmospheric refractive-index fluctuations upon the signal received by an astronomical instrument on the ground. Those lectures emphasized results for a Kolmogorov spectrum of turbulence with a large outer scale, and with relatively simple models of the vertical structure of turbulence strength and wind velocity. 'The measurements upon which the model is bissed have typicially been done at tenuporal and spittial scales of relevance to smatl ground-bised telescopes in the visible. An important question rentuins concerning the sensitivity of the quality of the images from a large-diameter ground-based telescope to the extrapolation of the turbulence model into regions of temporal and spatial separations that have not been measured.

For astronomicai purposes, the goal of creating atmospheric models is to make it possible to calculate the behavior of a large-diameter ground-based telescope with an adaptive-optics system; in particular, to calculate that behavior from measurements made with much smaller systems. $A$ measureine'nt of $r_{0}$ alone will not allow such an extrapolation. 'The observable we calculate here is complicated, and will be defined later, but its purpose is to provide a quantity that can be usefully extrapolated to large telescopes. This observable obtains information about larger spatial scales by using long-time correlations and the "frozen wind" (Taylor) hypothesis.

In the present paper, we treat two turbulence spectra: the Kolmogorov model 
and a "random walk" model suggested by Bester et al.(1992) to explain their observations with an infrared interferometer. We also couple both turbulence models with complex vertical structure in turbulence strength and wind velocity.

Bester et al. suggest that measurement.s taken with the Ihfrared Stellar Interferometer on Ml. Wilson show behavior which does not currespond to a kolmogorov spectrum with uniform wind velocity (13ester et al., 199\%). They proposed a model based on refractive index fluctuations with plyysical sizes distributed according to random walk statistics. 'This model was intended as a counter-example to the usual models. Bester et al. provide references for other experiments which may have shown non-kiolmogorov behavior. The "random walk" model essentially results in a power-law spectrum but with a different power-law index than kolmogorov.

The power-law index of the spectrum of measured plase variations due to fluctuations in the refractive index of the atmosphere is critical to the designers and users of adaptive opticial systems. The system designed to a spectrum which underestimates the relative strength of high frecuency phase fluctuations relative to the strength of those at low frequencies may have a larger residual wavefront phase error than expected after compensation. The situation is similar for systems which are designed to compensate at one wavelength, but primarily observe at another, since the predicted compensation error might greatly differ from the actual crror.

The outer scale of turbulence describes the scale at which the power-law ceases to be relevant. That is, it is the largest spatial scale at which energy enters the "turbulent cascade". At wavenumbers smaller than the inverse of the outer scale the power spectrum of phase fluctuations should show a plateau, unless other non-turbulent processes induce temperalure fluctuations in the atmosphere (and hence refractiveindex fluctuations). The value of the outer scale is a much debated item, with most measurements running from a few meters to 100 meters, and sometimes much larger(Bester el ial., 1092; Niglitengalle and Buscher, 1991; Coulman and Vernin, 1991; Colavita et al., 1987). It appears to be variable not only with site, but in time, with widely different values estimated at the same site at different times.

The significance of a small outer scale to adaptive optical systems will depend on just how small it is. Even an outer scale of 20 meters is small compared to the separation of elements in large interferometric arrays such as the VLT and the Keck I plus Keck II system, so that signals from system subclements would be completely decorrclated.

\section{Model Descriptions}

Let the power spectrum of refractive-index fluctuations in the atmosphere be designated by $\Phi(\kappa)$, where $n$ is a three-dimensional spatial wavevector. For the Kolmogorov model $\Phi$ is given by

$$
\Phi(\kappa)=0.033 C_{n}^{2}\left[\left(\frac{.071}{1.0}\right)^{2}+\kappa^{2}\right]^{-11 / 0},
$$

where $C_{n}^{2}$ is the strength of fuctuations and $L_{0}$ is the outer scale of turbulence. The numerator 1.071 in the outer-scale term was chosen to enable comparison to some 
other published results. In the literature, the value of the numerator is often cliosen to be either 1 or $2 \pi$, so comparison of outer scale values requires keeping the specific form of the numerator in mind.

For the "random walk" model, we have used a form similar to the Kolmogorov spectrum for the spatial power spectrum

$$
\Phi(\kappa)=R\left[\left(\frac{1.071}{L_{0}}\right)^{2}+\kappa^{2}\right]^{-3 / 2} .
$$

IIcre $R$ is a constant that sets the absolute level of the spectrum.

$\Lambda t$ this point we can calculate the beliavior of phase fluctuations for the light from a star to a point on the ground. Most readers will be familiar with derivations of the spectrum or structure function of these pliase fuctuations. Instead of these more familiar functions, we will use the Allan variance, a function that is used more often in interferometric systems than in imaging systems.

$A n$ interferometer with elements separated by a baseline 13 measures the phase differences $\delta \phi(B, t)=\phi(B, t)-\phi(0, t)$ between its elements. The $\Lambda$ llan variance of phase difference fluctuations is

$$
\sigma_{A}^{2}(\tau, B)=\left\langle\left[\frac{1}{\tau} \int_{t_{1}+\tau}^{t_{1}+I_{\tau}} \delta \phi(B, t) d t-\frac{1}{\tau} \int_{t_{1}}^{t_{1}+\tau} \delta \phi(B, t) d t\right]^{2}\right\rangle,
$$

where the $\langle\ldots\rangle$ represents averaging over all the intervals of length $\tau$ in the total measurement time. By assuming the Taylor hypothesis of "frozen flow", we can connect time intervals $\tau$ to spatial separations $\rho$ by $\rho=v \tau$. The Allan variance for a Kolmogorov spectrum and a specific clioice of baseline $B$, outer scale, wind profile, and $C_{n}^{\prime}$ profile, is shown in Figure 1.

The long-time asymptole of the Allan variance in Figure 1 represents atmospheric behavior at large spatial separations: in this case greater than $4 \mathrm{~m}$. Therefore the slope of this asymptote, denoted by $\beta$, is a measure of large-scale atmospheric behavior, the behavior that is of most relevance to large-diameter telescopes, or large-baseline interferometers. We will use $\beta$ as the parameter of interest to be calculated for various atmospheric models. The definition of "long time" is not always so clearly defined as in Figure 1, so we have to define the time at which we measure $\beta$. Bester et al. measured $\beta$ at times of about 10 s(Bester et al., 1992). We present here results for $\beta$ at $10 \mathrm{~s}$ and at $60 \mathrm{~s}$.

In order to calculate the Allan variance, and hence find $\beta$, we first express the power spectrum of temporal fuctuations in phase difference $W_{\delta \phi}(f)$ under conditions in which the velocity, the outer scale, and the atmospheric strength of fluctuations could vary. For the Kolmogorov model the approximate answer (we assume difraction terms are negligible) is given by

$$
\begin{aligned}
W_{\delta \phi}(f)= & 5.211 k^{2} \sum_{j=1}^{N} C_{n}^{2}\left(z_{j}\right) \Delta L_{j} v_{j}^{-1}\left\{\frac{\Gamma\left(\frac{1}{2}\right) \Gamma\left(\frac{1}{3}\right)}{\Gamma\left(\frac{11}{6}\right)} \beta^{-8 / 3}\right. \\
& \left.-2 \frac{\Gamma\left(\frac{1}{2}\right)}{\Gamma\left(\frac{11}{6}\right)} \cos \left(\frac{2 \pi \int B \cos \theta_{j}}{v_{j}}\right)\left[\frac{a}{2 \beta}\right]^{4 / 3} K_{4 / 3}(a \beta)\right\} .
\end{aligned}
$$




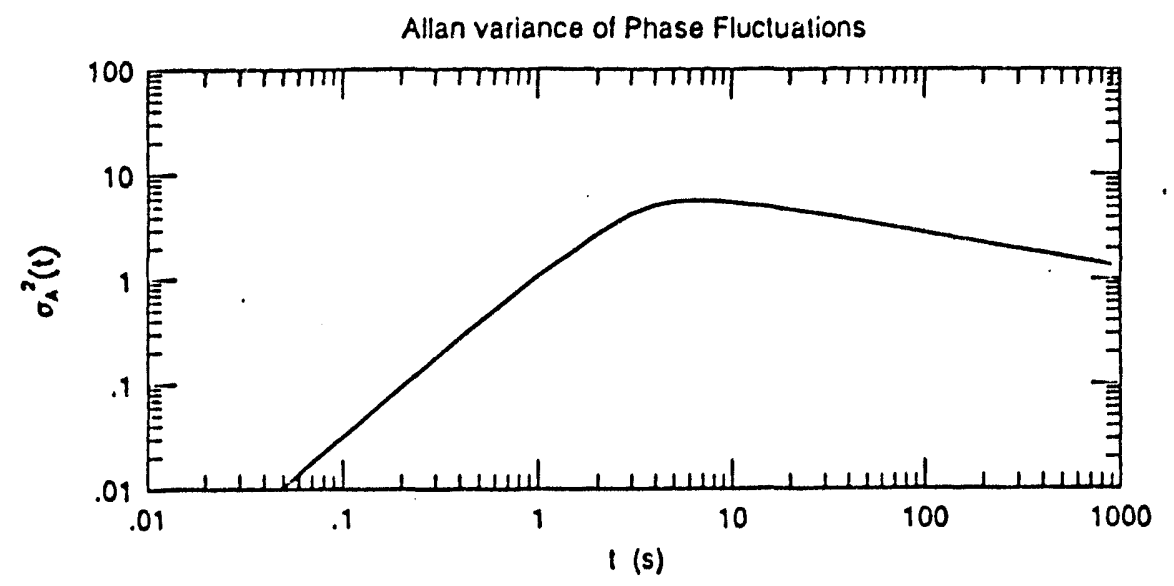

Fig. 1. Allan variance of pliase difference fluctuations for a pair of telescopes scparated by a baseline of $t$ meters. The aympectic form is $0^{2}(1) \sim t^{3 / 3}$ for bliall arguinesuly and $o^{2}(t) \sim t^{-1 / 3}$ for intervals where ut exceceds the laseline. For thir example the spectruin is Kolniogurov with $l_{0}=10 \mathrm{kin}$. We have takell $v=1 \mathrm{~m} / \mathrm{s}$ at all altitudes; therefore the Allall variance is independent of the $C_{1}^{2}$ profile.

where $\beta^{2}=\left(\frac{2 \pi l}{v_{j}}\right)^{2}+\left(\frac{1.071}{l_{0 j}}\right)^{2}, a=B \sin O_{j}$ and $K_{4 / 3}(\alpha \beta)$ is the modified Bessel function. Note that the mediurn hiss been divided into $N$ phase screens each with its own $C_{n, 2}^{2}$ Lo, velocity $v_{j}$, wind angle reliative to the baseline $O_{i}$, and characteristic distance to the next sereen $\Delta l_{j}$. The watvenumber of the radiation is $k, \Lambda$ comparable expression can be derived for lla "random walk" model.

Given the isbove power spectrum, we cill cislculate the structure function and the Allan variallce:

$$
D_{\delta \phi}(\tau)=\frac{1}{\pi^{2}} \int_{-\infty}^{+\infty} W_{\delta \phi}(\omega) \sin ^{2}\left(\frac{\omega \tau}{2}\right) d \omega
$$

and

$$
\sigma_{\lambda}^{2}(r)=\frac{1}{\pi^{2}} \int_{-\infty}^{+\infty} W_{\Delta \phi}(\omega) \frac{\sin ^{4}\left(\frac{\omega r}{\pi}\right)}{\left(\frac{w r}{2}\right)^{2}} d \omega .
$$

We have numerically evaluated $\sigma_{\lambda}^{2}(\tau)$, and then found the slope $\beta$ at $\tau=10$ $s$ and $60 \mathrm{~s}$. We have used 20 atmospheric layers, allowing us to evaluate complex models based on real meatsurements of $C_{11}^{2}$ and wind as functions of altitude.

We have used profiles of $C_{11}^{\prime 2}$ and $v$ from various experiments. The profiles are shown in Figure 2, where the dilferent experiments are labeled as follows: $\Lambda$ set of profiles denoted by $M K$ is from the Mituna kea site(Olivier et al., 1003). The $M K G$ profiles are the same as the MK profiles except that the ground-level $v$ has been reduced from 3 to $1 \mathrm{~m} / \mathrm{s}$. The II-V profiles coine from llufragel(1971) and Valley(1980). The PdM profiles come frorn ineasurernents on the Pic du Midi(llecquet and K'laus, 1989). T!se Mauna Kea and Pic du Midi profiles were measured at night 


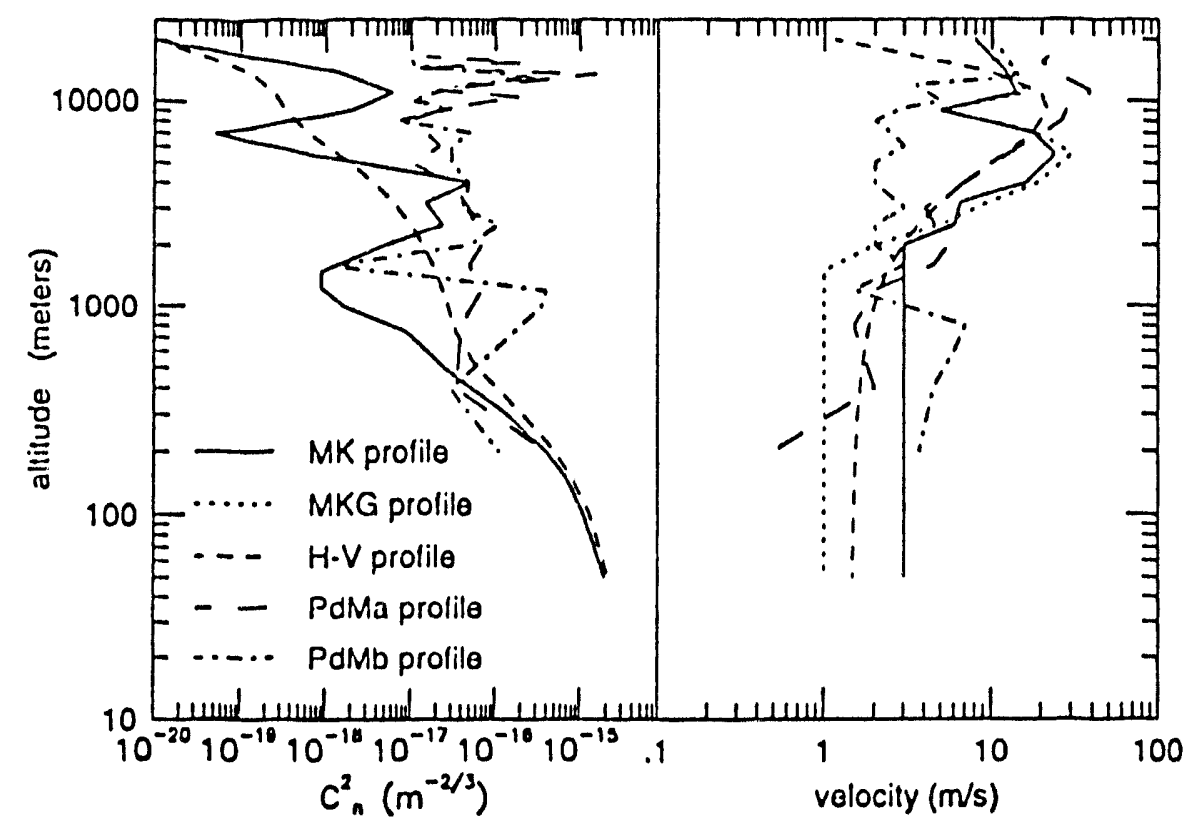

Fig. 2. Profiles of $C_{11}^{2}$ and wind opeal fur five dilferent nighlit-lime cases: MK = Mauna kea(Olivier

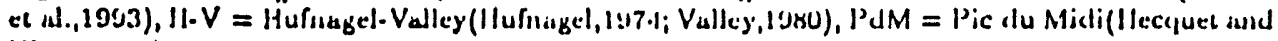
Klaus,1089).

and the flufnagel-Vislley inodel wiss scilled to be reppresentiative of good secing condltions. None of the models is precisely represcntative of the conditions encountered at Ml. Wilson; lowever, it is hoped that some verisimilicude is attitined.

The values we obleain for $\beta$ are dependent on the values of lie angles, $o_{j}$, between the bisseline of the interferometer and the wind velocity, which maty be a function of altitude. For the $M K, M K C^{\circ}$, and II.V profiles we have takell all $v_{j}$ to be $15^{\circ}$. For the PdM profiles we have assumed that the interferometer bascline were oricnted North-South, and we have: tatien the measured profile of wind velocity(llecquet and kilitus, 1980).

\section{Results}

We summarize our results in Figure 3. The loft panel shows the measurements of Bester et. al. for $\beta$ at 10 s. The other tiro panels show our results for $\beta$ at two diflerent times, its a function of outer scide to. 'The model results at 10 y show that both liolmogorov and "random walk" models give acceptiable values, provided that the profiles and outer scale are unknown. Even if tlie outer scale were known, different profiles create a substantial variation in $\beta$ at $10 \mathrm{~s}$ for both types of spectrum.

On the other hand, at $60 \mathrm{~s}$ the values of $\beta$ are much more definitive measures of the model characteristics. If the outer scale is $15 \mathrm{~m}$, then extrapolating to large 


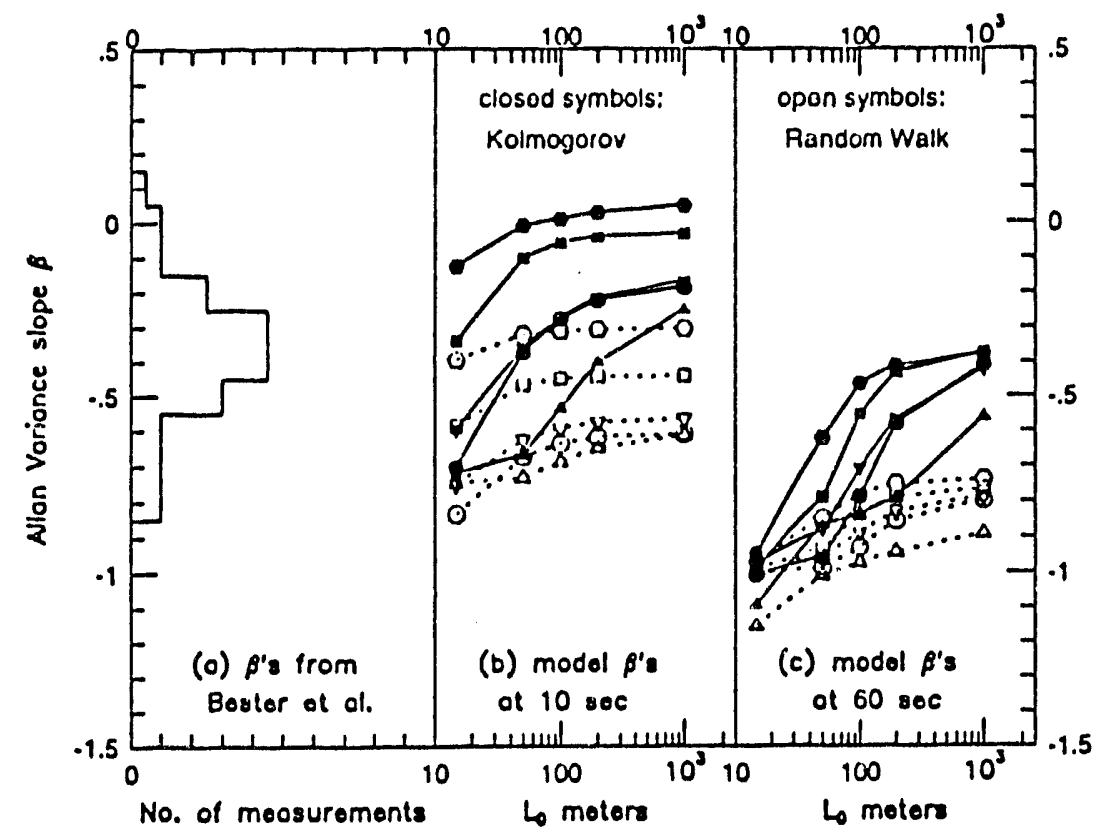

Fig. 3. Allan-variance slope $\beta$. (a) Measurements of Bester el a. (1992) with an infrared interferometer with baseline $f$ in and time separations of about 10 s; (b) Model results at 10 s as a function of outer scide L.o; (c) Model results at bU s. Dilferent symbuls represent different sets

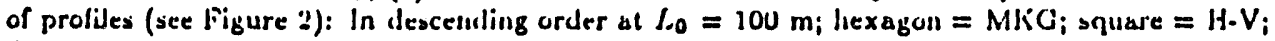

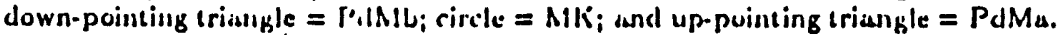

separations gives a plateau regardless of the power law in the small-scale region. $\Lambda \mathrm{s}$ a result the values of $\beta$ cluster around -1 for all models. If the outer scale is $1000 \mathrm{~m}$, then the power-law index largely delermilles $\beta$, and the Kolmogorov and "random walk" models are clearly distinguished. Al intermediate outer sciales the behavior for the two spectra is distinguishable for most proliles. It appears that if measurements of $\beta$ could be made at $60 \mathrm{~s}$, along with reisonable measurements of profiles, models could be usefully distinguished.

Unfortunately, at longer time intervals the chance of seeing contamination of the frequency spectrum from non-lurbulent processes increases. The apparent signature of gravity waves in the atmosphere is seen in some datit of Bester et al.

We have studied the differences in the 60-s $\beta$ from the different profiles and have concluded that the differences cant be attributed to differences in wind speed. Specifically, increasing wind speed results in a clecrease in $\beta$, and vice-versa. In complicated profiles, one must consider the weighted wind speed. For exanple, if the wind near the ground decreased while the wind at altitude increased, the effect will depend on the relative sizes of $C_{11}^{*}$ at the two altitudes. 


\section{Conclusions}

We have calculated the slope of the Allan variance for the fluctuations in the difference in phase between two elements of an interferometer. We used model atnospheres with realistic values of wind, refractive-index variations, and outer scale. We did so for the standard kolmogorov model of turbulence and for the "random walk" model suggested by Bester et al. Ilow well one can distinguish between spectral models seems to depend upon being able to measure the Allan variance for time intervals $\mathrm{cn}$ the order of one minute. We do find that a Kolmogorov model combined with some experimental profiles will produce values of $\beta$ at time intervals of 10 seconds which are comparable with those measured by Bester et al. Measurements at $60 \mathrm{~s}$ would distinguish models more clearly, and would place the effective spatial scales being measured closer to those of interest in large-diameter telescopes and interferometers. These measurements would be made significantly more useful for adaptive-optics-system design, tcst, and evaluation if more simultaneous measurements of relevant atmospheric quantities were made.

\section{Acknowledgements}

This research was supported in part by the Institute of Geophysics and Planetary Physics at the Lawrence Livermore National Laboratory (IGPP/LLNL), under US Department of Energy Contract 110. W-7405-ENG-18 to the University of California. We are grateful for a grant from the W.M. Keck Foundation. L.W.B. would like to thank the National Science Foundation, the Advanced Studies Institute Directors, and IGPP/LLNL for financial support to attend the summer school. We also thank the ISI experimenters, particularly C.II. 'Townes and Manfred Bester, for patient explanations ind for making data available to us.

\section{References}

M. Bester, IV.C. Danchi, C.G. Degiacomi, L.J. Greenhill, and C.II.Townes, 1992, "Atmospheric Fluctuations - Empirical Structure Functions and Projected Performance of Future Instruments," Astrophys. J., 392, 357-374.

M.M. Colavita, M. Shao, and D.II. Staelin, 1987, " $A$ tmospheric phase measurements with the Mark III stellar interferometer," $A p p l$. Opl. 26, 1106-1112.

C.E. Coulman and J. Vernin, 1991, "Significance of anisotropy and the outer scale of turbulence for optical and radio seeing", Appl. Opt., 30, 118-126.

J. Hecquet and V. Klaus, 1989, "Comparison of optical measurements of seeing and calculations based on radiosonde data," Astron. Astrophys., bf 225, 585-590.

R. E. Ilufnagel, 1974, Optical Propagation through Turbulence (Optical Socicty of America, Washington, D. C.).

N.S. Nightengale and D.F. Buscher, 1991, "Interferometric seeing measurements at the La Palma Observatory," Mon. Not. R. astr. Soc., 251, 155-166.

S.S. Olivier, C.E. Max, D.T. Gavel, and J.M. Brase, 1993, "Resolution Limits for Ground Based Astronomical Imaging Using Adaptive Optics," Astrophys. J., 407, 428-439.

G. C. Valley, 1980, "Isoplanatic degradation of tilt correction and slort-term imaging system," Appl. Opl. 10, 571-577. 

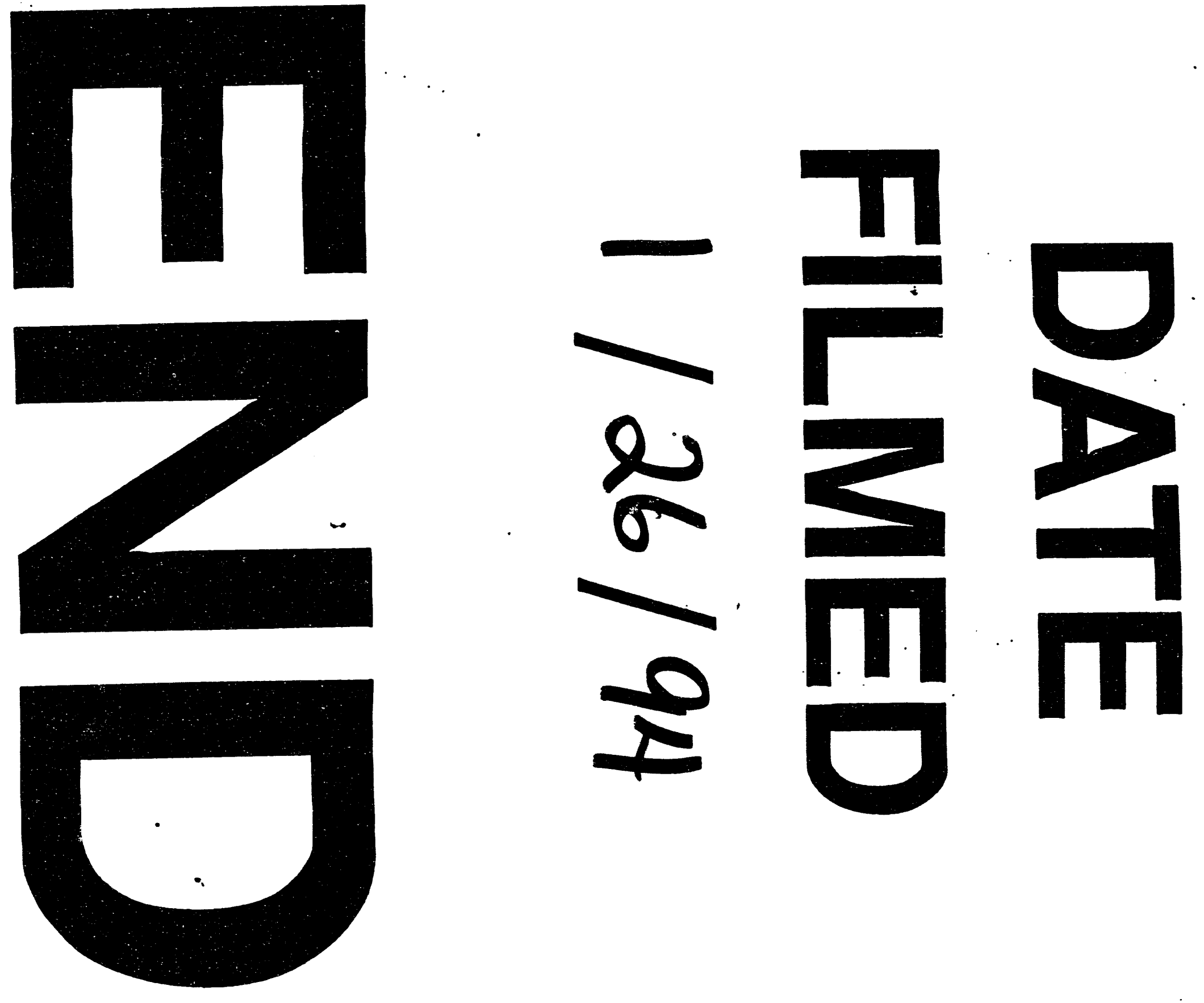
